\title{
CHILDREN AND ADOLESCENTS WITH RHEUMATIC DISEASES AND TUBERCULOSIS IN A TERTIARY HOSPITAL IN RIO DE JANEIRO - CASE SERIES
}

Lenita de Melo Lima ${ }^{1}$, Rafaela Baroni Aurilio ${ }^{1}$, Ana Alice Ibiapina Amaral Parente ${ }^{1}$, Adriana Rodrigues Fonseca ${ }^{1, \star}$, Claudia Stella Pereira ${ }^{1}$, Michely Alexandrino de Souza Pinheiro ${ }^{1}$, Belize Marques Barreto ${ }^{1}$, Sidnei Ferreira ${ }^{1}$, Flavio Roberto Sztanbok ${ }^{1}$, Marta Cristine Felix Rodrigues ${ }^{1}$, Maria de Fatima Bazhuni Pombo Sant'Anna ${ }^{1}$, Clemax Couto Sant'Anna ${ }^{1}$

1.Universidade Federal do Rio de Janeiro, Rio de Janeiro (RJ), Brazil.

*Corresponding author: adrirfonseca@gmail.com

\section{BACKGROUND}

The risk of illness due to tuberculosis (TB) is increased in patients with rheumatic diseases due to immunologic deregulation and to immunosuppressant or biological therapy, especially in a country endemic to the disease such as Brazil.

\section{METHODS}

Descriptive, observational and retrospective study, with the objective of describing a case series of children and adolescents who developed TB in the course of rheumatic diseases in a university hospital. Patients aged $0-18$ years with autoimmune rheumatic diseases, followed in a TB referral center and diagnosed with active TB in the last 15 years were included. The TB diagnostic was based on the Brazilian National Ministry of Health diagnostic scoring system, radiographic findings, bacilloscopy and rapid molecular test for tuberculosis (RMT-TB). The data were collected after ethical approval (No. CAAE45099121.3.0000.5264).

\section{RESULTS}

Seven cases with TB diagnosis, 2 (28.57\%) extrapulmonary forms (EPTB), of skin and peritoneum and $5(71.42 \%)$ in pulmonary forms (PTB). The median age in diagnosis was 117.7 months (73-161), male majority (4-57,14\%). The most frequent rheumatic disease was juvenile idiopathic arthritis (4-57.14\%), followed by systemic lupus erythematosus (2-28.57) and juvenile dermatomyositis (1-14.29\%). Corticosteroid was the most used drug (5/7-71.42\%), followed by methotrexate (3/7-42,85\%) and hydroxychloroquine ( $2 / 7-28.57 \%)$, besides cyclosporine $(1 / 7-14.28 \%)$ and adalimumab (1/7 - 14.28\%). All patients presented signs or symptoms of TB. All had high probability (superior to 30 ) clinical-radiological scores for active TB. The tuberculin skin test reaction was positive in $1 / 6(16.66 \%)$ patient, negative in 3/6 (50\%) and not performed in $2 / 6$ (33.33\%). Chest radiographic changes in the PTB cases were: hilar lymph gland enlargement in $1 / 5$ (20\%), miliary pattern in $2 / 5$ (40\%) and alveolar opacifications in $2 / 5$ (40\%). Bacilloscopy and culture were negative in all patients; RMT-TB was positive in all cases with EPTB and in 1 with PTB.

\section{CONCLUSION}

The patient clinical evaluation and the clinical scoring system were relevant to the diagnostic in most patients, and the RMT-TB proved to be important in cases of EPTB. The findings highlight the value of clinical and radiological diagnosis in TB cases and the necessity of considering the disease as an important adverse event in patients with rheumatic diseases.

\section{KEYWORDS}

Children, Adolescents, Tuberculosis, Rheumatic diseases. 